\title{
Evidence suggesting that domestic chicks should not be used in "imprinting" research: Variability in performance during "imprinting's" critical period
}

\author{
L. JAMES SHAPIRO \\ Avian Behaviour Laboratory, University of Manitoba, Winnipeg, Manitoba R3T 2N2, Canada
}

\begin{abstract}
Three experiments were conducted under tightly controlled conditions in order to provide empirical evidence that chicks should not be used in research studying the formation of attachments in precocial birds. In a pilot study, a main study, and a replication, several different periods of time were found within and between experiments during which an attachment seemed to be formed between a chick and the training object. No statistically significant differences between any treatment groups were obtained in the main study or the replication, indicating a lack of reliable (i.e., repeatable) performance on the part of the subjects.
\end{abstract}

Most "imprinting" research has been done with chicks and ducklings. One of the problems associated with the use of domestic chicks in such research is that these organisms do not exist in the natural environment. Hence, there is no natural context for the domestic chicken's behavior. The domestic chicken is a cardboard organism. It was pressed together by the culinary, sports, agricultural, and religious interests of men (Zeuner, 1963) and is held together by his economic needs (Wood-Gush, 1971). There are no domestic chickens living in a natural environment that have evolved on their own without any assistance from human beings during the evolution of the earth. Researchers using chicks, therefore, have no organism that they can observe in its natural environment in order to know what is normal behavior and to which they can later generalize their laboratory-generated results.

Another problem associated with the use of domestic chicks in "imprinting" research is their variability in performance during the conduct of such research. For many years, the "imprinting" literature has reported a variety of results that have often been contradictory with respect to the performance of domestic chicks. Within laboratories conducting long-term research projects involving these subjects, the results are often unreliable. Between laboratories the unreliability of chicks increases.

The author would like to thank N. R. Remley and S. H. Evans for their extensive comments made on an earlier draft of this manuscript. The research reported in this article was originally done at Texas Christian University and was supported, in part, by the TCU Research Foundation through the Institute for the Study of Cognitive Systems. Portions of this article were used by the author in partial fulfillment of the requirements for the MA degree. Requests for reprints should be sent to L. James Shapiro, Avian Behaviour Laboratory, Department of Psychology, University of Manitoba, Winnipeg, Manitoba R3T 2N2, Canada.
An empirical demonstration of the lack of stability exhibited in the performance of chicks could be made if a tightly controlled situation using domestic chicks was repeatedly used to evaluate a more or less "accepted" aspect of the phenomenon known as "imprinting." Such a situation is the laboratory constructed by Shapiro (1970) so that he could study the formation of attachments in precocial birds. The critical period is, more or less, an accepted aspect of imprinting. It is one of the original attributes of imprinting put forth by Hess (1959), Lorenz (1935/1957), and a host of others (for a review of this literature, see Bateson, 1966; Hess, 1973; Sluckin, 1965; Smith, 1969) and is usually thought of as being a part of the process associated with forming an attachment in a precocial bird (but see Shapiro, 1980, for other possibilities).The studies described in this article are reported in order to provide empirical evidence that the use of chicks as subjects in "imprinting" research is not advisable.

\section{GENERAL METHOD}

\section{Subjects}

White Leghorn (Gallus gallus) eggs were incubated in a Lyon A475 glass display incubator (Lyon Electric Company, San Diego, California). The relative humidity and temperature of the incubator were maintained at $62 \%$ and $37.8^{\circ} \mathrm{C}$, respectively. The eggs were turned by hand three to five times each day. During the 21-day incubation period, the room housing the incubator was kept well lighted $24 \mathrm{~h} /$ day. When hatching commenced, the lights were turned off. The exact time of each hatching was recorded with the assistance of a small flashlight. A subject was considered hatched when it was completly separated from its shell. The experimenter gave no assistance to any subject separating from its shell. No direct lighting fell on the subjects at any time during hatching or prior to training and testing.

Immediately after hatching, each subject was placed in a randomly chosen compartment of a specially constructed brooder (Shapiro, 1970) and individually housed in heat- 
controlled compartments $31.1 \times 6.4 \times 20.3 \mathrm{~cm}$, with sides, ceiling, and $.63-\mathrm{cm}$ wire-mesh flooring painted a flat black. Placement in a compartment also mounted to assignment to a particular group. Subjects were fed a 1:3 ratio of grit and Purina Chick Startena. An ad-lib supply of food and water was maintained. The construction of the brooder was such that the experimenter's hand never had to enter the subjects' quarters except to remove the subject for experimentation and to replace it afterward. With subjects kept for a long period of time, replacement of food took place in complete darkness or while the subject was being trained or tested. The temperature of the brooder was independently monitored by separate thermometers randomly placed within the brooder itself, and the thermostat was set between $28.9^{\circ} \mathrm{C}$ and $32.2^{\circ} \mathrm{C}$, depending upon the number of subjects in the brooder at the time and the readings on the thermometers.

\section{Apparatus}

The apparatus has been described in detail elsewhere (Shapiro, 1970). Briefly, the apparatus used was a circular runway $30.5 \mathrm{~cm}$ wide, $1.52 \mathrm{~m}$ in diameter (measured from the outside walls), and $3.83 \mathrm{~m}$ in circumference at the center of the runway. The runway boundaries were formed by $30.48-\mathrm{cm}$-high walls of clear Plexiglas; the floor was of $1.9-\mathrm{cm}$ plywood. A red or a white ball, $21.6 \mathrm{~cm}$ in circumference, hung $7.6 \mathrm{~cm}$ above the runway floor, suspended from an elevated arm radiating from the center of the apparatus. The arm suspending the ball moved at the rate of $30.48 \mathrm{~cm} / \mathrm{sec}$ and had the following temporal pattern of progression: 4-sec run, 8-sec pause; 4-sec run, 8-sec pause; 6-sec run, 30 -sec pause. This sequence was repeated once each minute throughout each session. The resulting journey of the ball was $5 / 6$ th of a round trip in each minute's sequence. This rate of movement insured that the ball did not describe the same locality pattern more of ten than once every $6 \mathrm{~min}$.

By means of photoelectric cells and light sources embedded in Plexiglas plates external to the runway, the number of seconds each subject spent within $15.24 \mathrm{~cm}$ of any side of the ball was automatically recorded. The Plexiglas plates holding the photoelectric cells and light sources were attached to the same rod that held the arm from which the ball was suspended. The entire apparatus, being welded to the same center pole, rotated as a unit.

The apparatus was placed inside a $2.44 \times 2.44 \times 1.98 \mathrm{~cm}$ sound-deadened chamber made of double-walled Celo-tex and insulated with a sound-attenuating substance. Even illumination was provided by a three-tube, circular $40-\mathrm{W}$ fluorescent fixture A one-way vision window was placed in one wall. Adjacent to this window was a console containing all the switches necessary for operating the incubator and the equipment in the experimental chamber. Two Standard Electric timer clocks of $10 \mathrm{~min}$ range were wired into the console and provided the means of reading the cumulative times indicated by the photoelectric cells.

\section{Procedure}

In each experiment, subjects were randomly assigned to a group. The groups represented different periods of time. When a particular subject in any group reached the age associated with that group, it was removed from its brooder compartment in the dark in such a way that the experimenter's hand covered its head. The subject was then carried into the experimental chamber and placed on the runway floor beside the training object, facing in the direction of motion. Either a red or a white ball was presented to each subject. The presentation of the balls was balanced over subjects. After $1 \mathrm{~min}$, the motor was started and the object began its journey. The subject was returned to its home cage $25 \mathrm{~min}$ after the training session began. A 25-min preference test was administered some time after the training session, the exact period of elapsed time depending upon the experiment involved. During the preference test, the same procedures were used as those previously described for the training session, except that now both the red and the white balls were in the apparatus, suspended $180 \mathrm{deg}$ from each other. The subject was placed halfway between the two balls at a 90-deg angle to each of them. The unfamiliar ball was placed so that it moved away from the subject.

\section{PILOT STUDY}

A pilot study was conducted to determine if subjects could discriminate between a white ball and a red ball, to evaluate the strength of locomotion as a function of maturation, and to canvas a wide range of ages to determine if there were any peak periods during which an attachment to the stimulus object seemed to be formed and which could later be subjected to repeated testing.

\section{Method}

Subjects and Apparatus. Sixty-five subjects were used in this study in the apparatus previously described.

Procedure. Five subjects were randomly assigned to each of 13 groups. The groups represented the following periods of time, in terms of hours since hatching: (1) $0-6$, , (2) $6-12$, (3) $12-18$, (4) $18-24$, (5) $24-30$, (6) $30-40$, (7) $40-50$, (8) $50-60$, (9) $60-80$, (10) $80-100$, (11) $100-120$, (12) $120-150$, (13) $150-180$. Twelve hours after the training session ended, each subject was given a 25-min preference test. In this study, a second preference test was also administered $180 \mathrm{~h}$ (7.5 days) after the first preference test. The purpose of the second preference test was to determine if maturational improvement in ability to locomote was capable of influencing the results of forming an attachment on subsequent preference tests. It was assumed that if maturational improvement could account for better following among subjects, then there should be more following among those subjects trained at a later age and also better following in subjects given a second preference test.

\section{Results}

The results of the first preference test revealed a lack of any attachments formed during the first $24 \mathrm{~h}$. Subjects in the 24- to 30-h and in the 60- to 80-h age groups, however, spent almost twice as much time following the training ball as they did following the nontraining ball. These peaks are clearly represented when difference scores (time spent following the training ball minus time spent following the nontraining ball) are used as the the response measure. These data are represented in Figure 1. The peaks obtained from these two groups strongly suggest different periods of time during which an attachment is formed in White Leghorn chicks.

The results of the first preference test also indicated that the subjects could discriminate between the red and white balls used as the stimulus objects. This conclusion is supported by the fact that some of the subjects clearly showed a preference for the training ball during the preference test. The fact that this preference was demonstrated regardless of the color of the training ball indicates that an attachment was formed and that it was not just a manifestation of innate preferences of a particular color.

The results of the second preference test revealed that only the subjects in the 60 - to 80 -h age group maintained the strength of their following responses. These data are also represented in Figure 1. In most groups, 


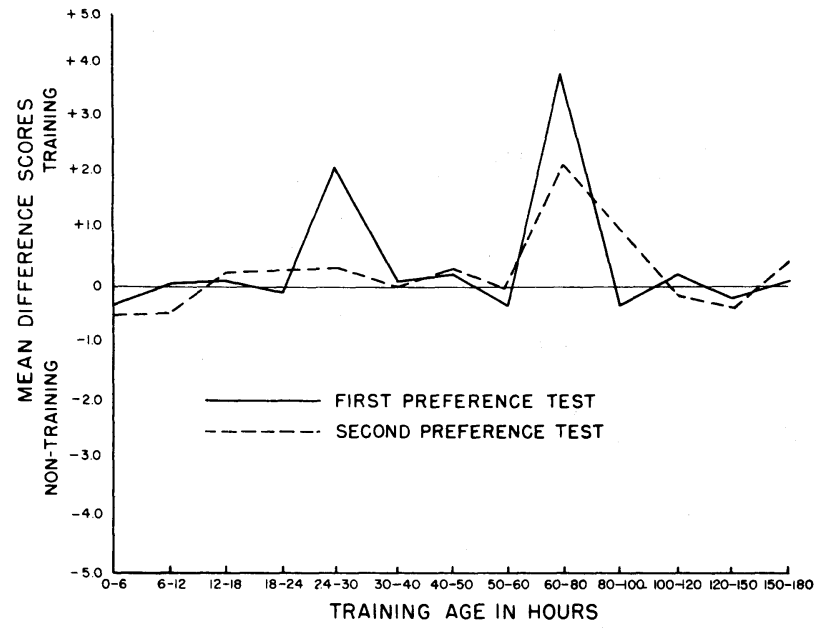

Figure 1. Difference scores between mean time (in minutes) spent near the training and nontraining balls during the first and second preference tests in the pilot study.

the mean following times during the first preference test were as large as or larger than the mean following times during the second preference test. These results indicate that maturational improvement in ability to locomote did not influence the formation of an attachment on the preference tests. It was concluded that locomotion as a function of maturation is not a relevant parameter of the following response. In other words, better following during a preference test should not be attributable to maturational improvement.

The pilot study demonstrated that the subjects could discriminate between a white and a red ball. The study also indicated that maturational differences in locomotive ability do not importantly affect the following response within the age ranges under investigation. Finally, the occurrence of the two peaks in the first preference test seemed to indicate that the first $80 \mathrm{~h}$ of age would be the most productive to investigate in subsequent experiments.

\section{MAIN STUDY AND REPLICATION}

In these experiments, the second preference test was omitted, the length of time between training and testing was modified, and only the first $80 \mathrm{~h}$ of age were studied.

\section{Method}

Subjects. In both the main study and in the replication, 104 White Leghorn eggs were incubated. Of the 91 subjects that developed full term in the main study, 81 , or $89 \%$, hatched. Of the 94 subjects that developed full term in the replication, 90 , or $95.7 \%$, hatched. No subject died during training or testing in either the main study or the replication.

Apparatus. The apparatus used in the pilot study was used in the main study and in the replication.

Procedure. Eight subjects were randomly assigned to each of eight groups. The groups represented the following periods of time, in terms of hours: (1) $6-12$, (2) $12-18$, (3) $18-24$, (4) $24-30$, (5) $30-42$, (6) $42-54$, (7) $54-66$, (8) 66-78. Only one preference test was conducted, and it occurred $24 \mathrm{~h}$ after each subject's training session. The second preference test was omitted because it had already been shown in the pilot study that maturational improvement in ability to locomote was not capable of influencing the results. The duration of time between the training session and the preference test was lengthened to facilitate the scheduling of the subjects' training and preference sessions.

\section{Results}

Difference scores (time spent following the training ball minus time spent following the nontraining ball) were used as the response measure in both the main study and the replication. In the main study, strong periods of following were associated with the 12- to 18-, 24- to 30-, 42- to 54-, and the 54- to 66-h age groups. These data are presented in Figure 2.

The two peak periods observed in the pilot study (Figure 1) are only partially seen in the two subsequent studies. The earlier peak, associated with the 24- to 30-h group in the pilot study, was found in the corresponding group of the main study, but not in the replication. The later peak in the pilot study had been associated with the 60- to 80-h group. On the basis of that result, the ages beyond $30 \mathrm{~h}$ were partitioned differently in the subsequent studies so as to yield smaller ranges in the region of this peak. Thus, the subsequent studies have two groups (54-66 $\mathrm{h}$ and 66$78 \mathrm{~h}$ ) that include parts of the period covered by the 60 - to 80 -h group in the pilot study. Examination of Figure 2 indicates that the 54- to 66 -h group shows some indication that an attachment has been formed in both subsequent studies, whereas the 66- to 78-h group shows evidence of forming an attachment only in the replication. Moreover, both subsequent studies show evidence of forming an attachment in the 42- to 54-h group, but neither group covering this period in the pilot study $(40-50 \mathrm{~h}$ and $50-60 \mathrm{~h}$ ) gave evidence of forming an attachment. A comparison of Figures 1 and 2 suggests that the second peak in the pilot study was shifted toward an earlier age and possibly spread out

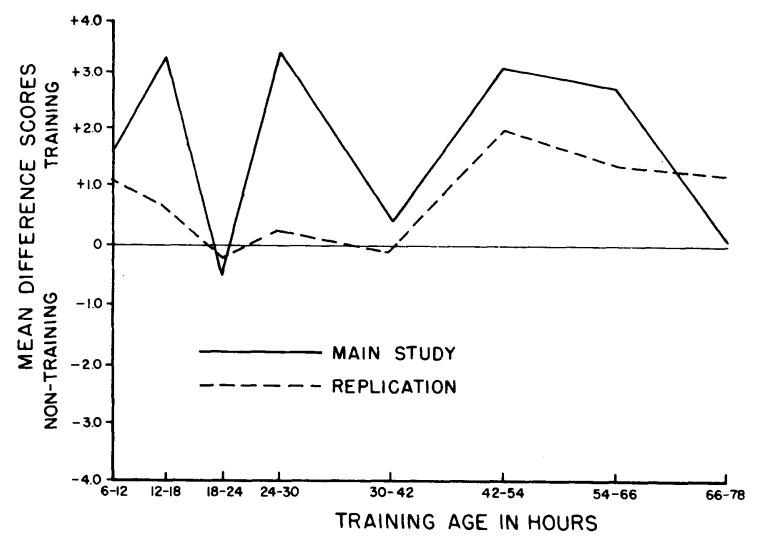

Figure 2. Comparisons of difference scores between mean time (in minutes) spent near the training and nontraining balls during the preference test in the main study and in the replication. 
over a larger age range. In other words, it was not replicated in the main study or in the replication.

Both the main study and the replication show evidence of a preference for the training object within the first $18 \mathrm{~h}$ but contradict the absence of this effect in the pilot study. In addition, the first peak periods in the main study and in the replication do not coincide.

A one-way analysis of variance, separately performed on the difference scores in the main study and in the replication, indicated no statistically significant differences $(p>.05)$ between any of the treatment groups in either of the studies. Harley's F MAX $_{\text {A }}$ test was used to test the appropriateness of the assumption of homogeneity of variance among the treatment groups in each study. The test indicated heterogeneity of variance among the treatment groups of the main study $\left[F_{\mathrm{MAX}}(8,7)=185.2, \mathrm{p}<.01\right]$. The same test also indicated heterogeneity of variance in the replication $\left[\mathrm{F}_{\mathrm{MAX}}(8,7)=16.4, \mathrm{p}<.05\right]$.

\section{GENERAL DISCUSSION}

Heterogeneity of variance, in conjunction with the graphic evidence of differences between groups, would suggest that the age variable did have an effect, but not a constant effect, for all subjects within each group, as is assumed by the analysis of variance model. It is this lack of consistency that prompts the suspicion that chicks are inappropriate subjects to be used in this type of research. Under the present tightly controlled conditions, one would expect that any effect exerted by one's independent variable would have a good chance of being replicated. Instead, the lack of any statistical significance in the main study and in the replication implies a lack of effects attributable to the independent variable (age) and militates against either a critical period hypothesis or a sensitive period hypothesis. On the other hand, one must suspect the organism used in these studies. The lack of replicability, the absence of statistically significant differences among the treatment groups, and the heterogeneity of variance provide a good demonstration of the lack of stability exhibited in the performance of chicks in this type of research. Taken together, the results provide empirical evidence that the use of chicks as research subjects in "imprinting" research is not advisable.

Strictly speaking, these results apply only to the concept of a critical period. The variability of chicken data in an investigation on critical periods need not imply comparable problems for chicken research on other imprinting-related topics. These results, however, should be of some concern to other researchers considering the use of chickens in their "imprinting" research and should stimulate a reconsideration of the type of subject one should use in research of this nature. It can be argued that other researchers in the field have managed to produce very good data with chickens over the years. Whether these data are generalizable to the number of precocial birds that are supposed to "imprint" is debatable.

\section{REFERENCES}

Bateson, P. P. G. The characteristics and context of imprinting. Biological Review, 1966, 41, 177-220.

HEss, E. Imprinting. Science, 1959, 130, 133-141.

HEss, E. Imprinting: Early experience and the developmental psychobiology of attachment. New York: Van Nostrand Reinhold, 1973.

Lorenz, K. [Companionship in bird life: Fellow members of the species as releasers of social behavior.] In C. H. Schiller (Ed. \& trans.), Instinctive behavior. New York: International Universities Press, 1957. (Originally published, 1935.)

Shapiro, L. J. Automation and experimental control in a laboratory for imprinting research. Journal of Comparative and Physiological Psychology, 1970, 73, 421-426.

Shapiro, L. J. Species identification in birds: A review and synthesis. In A. Roy (Ed.), Species identity and attachment. New York: Garland STPM Press, 1980.

Sluckin, W. Imprinting and early learning. Chicago: Aldine, 1965.

Sмiтн, F. V. Attachment of the young. Imprinting and other developments. Edinburgh: Oliver \& Boyd, 1969.

Wood-Gush, D. G. M. The behaviour of the domestic fowl. London: Heinemann Educational Books, 1971.

ZEUNer, F. E. $A$ history of domestic animals. London: Hutchinson, 1963.

(Received for publication October 1, 1980.) 